\title{
Valeur économique de la chasse au grand gibier. La méthode de transfert des bénéfices appliquée à la Région Aquitaine
}

The economic value of big game hunting. The benefit transfer method applied to the Aquitaine Region

\section{Hanitra Rakotoarison et Patrick Point}

\section{(2) OpenEdition}

\section{Journals}

Édition électronique

URL : http://journals.openedition.org/economierurale/3373

DOI : 10.4000/economierurale.3373

ISSN : 2105-2581

\section{Éditeur}

Société Française d'Économie Rurale (SFER)

Édition imprimée

Date de publication : 1 mars 2012

Pagination : 93-113

ISSN : 0013-0559

Référence électronique

Hanitra Rakotoarison et Patrick Point, « Valeur économique de la chasse au grand gibier. La méthode de transfert des bénéfices appliquée à la Région Aquitaine », Économie rurale [En ligne],

327-328 | janvier-mars 2012, mis en ligne le 01 mars 2014, consulté le 01 mai 2019. URL : http:// journals.openedition.org/economierurale/3373; DOI : 10.4000/economierurale.3373 


\section{Valeur économique de la chasse au grand gibier La méthode de transfert des bénéfices appliquée à la Région Aquitaine}

Hanitra RAKOTOARISON • Office national des forêts (ONF)

Patrick POINT • Centre national de recherches scientifiques (CNRS)

\section{Introduction}

$\mathbf{E}^{\mathrm{n}}$ France, le sanglier fait partie de la liste des animaux susceptibles d'être classés comme nuisibles ${ }^{1}$ (Code de l'environnement, article R. 427-6). L'évolution des tableaux de chasse nationaux est un indicateur de cette explosion démographique puisque ceux des sangliers ont été multipliés par 4,7 sur les vingt dernières années (ONCFS, FNC, 2010). Les autres espèces de grand gibier ont suivi cette trajectoire puisque les tableaux de chasse des cerfs ont été multipliés par 3,8 et ceux des chevreuils par 3,2 sur la même période. La prolifération du grand gibier crée des dommages agricoles estimés à environ 19,5 millions d'euros en 2005 au niveau national (FNC, 2006), des coûts liés aux collisions avec les automobiles estimés à 180 millions d'euros par $a^{2}$ (Saint-Andrieux, 2005) et des pressions importantes sur les jeunes plants forestiers ${ }^{3}$. Dans la Région Aquitaine qui est une zone à forte production agricole et forestière, les dommages importants du grand gibier créent des conflits entre chasseurs, sylviculteurs et agriculteurs. La croissance rapide des tableaux de chasse et l'importance des

\footnotetext{
1. Il appartient cependant au Préfet de chaque département de déterminer, parmi cette liste, les espèces classées nuisibles en fonction de la situation locale (Code de l'environnement, article R. 427-7).

2. Ce chiffre s'appuie sur des études économiques relatives aux coûts cumulés de la vie humaine, des blessés graves et légers ainsi que des dégâts matériels.

3. Les pertes économiques liées aux dommages forestiers sont encore difficiles à estimer, mais elles risquent de prendre de l'ampleur sans une régulation efficace de la population de cervidés.
}

dégâts économiques occasionnés sont d'autant plus préoccupants qu'on observe actuellement une baisse de l'exercice de la chasse. Le nombre de chasseurs français, estimé à 1,4 millions en 2005, diminue d'environ $2 \%$ à $3 \%$ par an (Scherrer, 2002). Cette évolution est liée au vieillissement des pratiquants, à l'augmentation des coûts de cette activité et à un changement de mentalité.

Face à cette situation, les politiques de gestion de la faune sauvage prônent actuellement pour la recherche d'un équilibre agro-sylvo-cynégétique (Code de l'environnement, articles L425-4 à L425-5). On peut approcher cet équilibre préconisé par la loi avec une analyse coûts-bénéfices (ACB) qui fait la somme des bénéfices de la chasse, des bénéfices de non-usage (liés à la présence actuelle et à la préservation future des animaux), moins l'ensemble des dégâts générés par le grand gibier ${ }^{4}$. L'agrégation de ces différentes valeurs économiques permet de donner ce que l'on appelle le bénéfice net social du grand gibier. Sa maximisation sous une contrainte liée à la dynamique de la population permet d'aider à la recherche d'une gestion optimale de la ressource.

Pour atteindre cet équilibre, les outils actuels de gestion du grand gibier sont basés sur les textes juridiques relatifs au prélèvement maximal de gibier et au plan de chasse (Code de l'environnement, L. 425-14). Ces instruments réglementaires sont difficiles à mettre en place en présence d'incertitude sur

4. La formalisation mathématique du modèle ACB avec une prise en compte de la dynamique de la population a été effectuée par Rakotoarison (2009). 
la taille de la population du grand gibier et ne sont pas adaptés en cas de surpopulation. Pour résoudre les conflits d'usage entre les acteurs économiques et mieux valoriser la ressource, une régulation avec l'aide des instruments économiques (taxe, subvention et marché de droit) pourrait être plus adaptée. Comment obtenir alors une valeur de référence fiable du bénéfice de la chasse qui est une activité récréative nonmarchande? Que nous enseigne les évaluations disponibles dans la littérature et comment peut-on estimer la valeur économique de la chasse au grand gibier pour les chasseurs, en l'occurrence dans la Région Aquitaine ?

L'objectif de cet article est de mesurer la valeur économique à la demande de chasse au grand gibier dans la Région Aquitaine. La difficulté de mesurer ce type de bénéfice est liée au fait qu'ils ne sont que partiellement monétarisés à travers les prix des bracelets de chasse et les baux de chasse. Cette absence de prix sur le marché est d'autant plus accentuée dans la Région Aquitaine par rapport au reste de la France que la chasse est basée sur le régime des Associations communales de chasse (ACCA) ${ }^{5}$. Ce régime stipule que les propriétaires forestiers rétrocèdent gratuitement leur droit de chasse au bénéfice des chasseurs de la commune sans contrepartie financière. Ainsi, particulièrement dans cette région, la demande en chasse repose sur les aspects sociaux, sportifs et récréatifs de cette activité. La méconnaissance de la valeur économique de la chasse rend toutefois difficile les décisions de gestion de la population cynégétique. Pour évaluer le bénéfice des activités de loisir, les économistes font appel aux notions de « Consentements à payer » (CAP), qui se traduisent par la somme monétaire que le

5. Le nouveau Code rural reconnaît le droit de nonchasse et permet aux propriétaires opposés à la chasse au nom de convictions personnelles de retirer leurs terrains des ACCA sans limitation de surface (article L.222-10 du Code rural). consommateur aurait donné pour exercer cette activité. Certaines études économiques font également référence aux « Consentements à recevoir » (CAR) qui expriment ce que le consommateur aurait accepté pour renoncer à cette activité. Ces deux notions mesurent la variation du surplus du consommateur retirée d'une amélioration ou d'une détérioration du bien-être à la suite d'un changement sur les conditions de pratique d'une activité récréative (Bonnieux, Desaigues, 1998).

Une approche très fine du bénéfice de la chasse supposerait la mise en œuvre d'un travail d'enquête spécifique mobilisant les techniques d'évaluation contingente, de choix multi-attributs ou de coût de déplacement. Le coût et la durée de mise en œuvre de ce type d'investigation conduisent souvent à y renoncer. Nous proposons de recourir ici à la Méthode de transfert de bénéfices (MTB) qui mobilise les résultats d'études déjà réalisées dans des contextes approchants. La première partie de l'article propose une brève synthèse des méthodes d'évaluation des biens et services non-marchands et expose les intérêts de cette méthode. La deuxième partie expose la démarche qui consiste à créer un méta-modèle à partir des études antérieures menées aux ÉtatsUnis. La dernière partie utilise ce modèle afin d'estimer le bénéfice de la chasse dans la Région Aquitaine.

\section{Les méthodes d'évaluation des biens et services non-marchands Intérêts des techniques de transfert de bénéfices}

\section{Les méthodes d'évaluation directes et indirectes}

Il existe deux grandes familles de méthodes pour mesurer les valeurs des biens et services non-marchands.

- Premièrement, il y a les méthodes indirectes qui sont des techniques de révélation des préférences du consommateur. Elles 
Figure 1. Classification des études référencées sur l'évaluation économique de la chasse au grand gibier

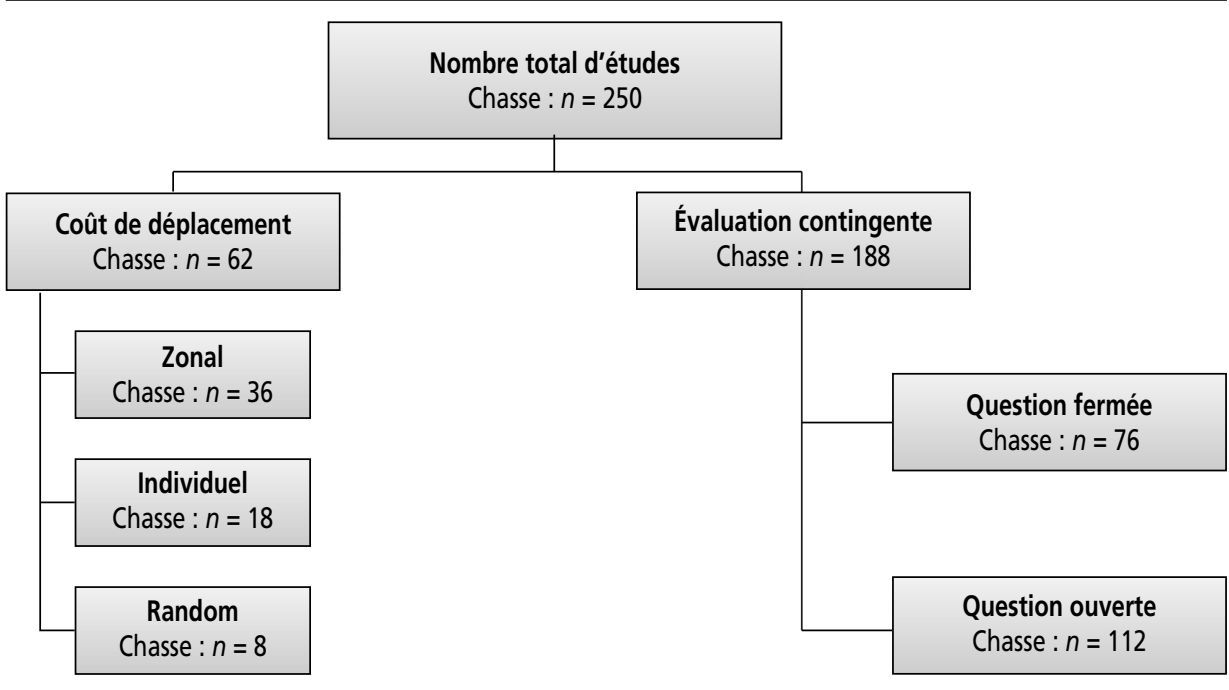

Note $: n=$ nombre d'études observées

Source : les auteurs

permettent de relier la participation à une activité récréative avec les dépenses engagées par l'individu. Dans cette famille, la Méthode des coûts de déplacement (MCD) est la plus fréquemment utilisée par rapport à d'autres méthodes indirectes comme la méthode de prix hédonique. Initiée par Hotelling en 1949, cette méthode estime le surplus du consommateur en dérivant une fonction de demande Marshallienne. Celle-ci relie le nombre ou le taux de fréquentation avec les prix (coût du voyage, prix de l'hébergement, droit d'entrée, coût d'opportunité du temps...). La MCD peut être appliquée à l'échelle de plusieurs zones géographiques (méthode zonale) ou à l'échelle individuelle, ce qui permet d'introduire les caractéristiques socio-économiques (âge, sexe, catégorie socioprofessionnelle...) dans la fonction de demande. La MCD random, reposant sur la théorie de l'utilité aléatoire, est particulièrement adaptée lorsque le chasseur peut choisir entre plusieurs sites ayant des caractéristiques différentes. Cette méthode peut être considérée comme une version de MCD plus sophistiquée car une modélisation des choix des sites précède celle de la fréquen- tation. Les MCD sont utilisées pour estimer des valeurs d'usage d'un actif environnemental : les activités récréatives, la consommation des produits ligneux et non ligneux...

- Deuxièmement, il y a les méthodes directes qui constatent les préférences des consommateurs en construisant un marché fictif à partir duquel les consommateurs expriment leur CAP/CAR pour une ressource environnementale ou une activité récréative. Parmi les méthodes directes, la méthode d'évaluation contingente (MEC) est la plus utilisée par rapport à l'analyse conjointe et la méthode des choix multi-attributs. Née des travaux de Ciriacy-Wantrup en 1947, celleci consiste à demander directement aux personnes cibles la valeur de leur CAP ou leur CAR pour une activité de récréation ou une modification des conditions de pratique de celle-ci. La personne enquêtée peut prononcer librement ses valeurs économiques (question ouverte) ou peut être guidée par des grilles de réponse (question fermée). Les MEC utilisent une courbe de demande Hicksienne compensée dans la mesure où on cherche à compenser l'individu pour qu'il conserve le même niveau d'utilité 
(Cummings et al., 1986). Le principal avantage des MEC par rapport aux MCD est leur possibilité d'estimer les valeurs d'usage et de non-usage, ce qui englobe les valeurs d'option, les valeurs d'existence et les valeurs de legs (Krutilla, 1967).

Comme la figure 1 le montre, les MEC ont été plus largement utilisées par rapport aux MCD dans la littérature sur l'évaluation économique de la chasse. Est-ce que ces choix méthodologiques conduisent à des mêmes valeurs économiques ? La méthode de transfert de bénéfices permet d'analyser cette question.

\section{Présentation des méthodes de transfert de bénéfices}

La Méthode de transfert de bénéfices (MTB) rentre dans une autre famille de méthodes d'évaluation des biens et services nonmarchands. Elle a fait son apparition vers les années 1980 (Sorg, Loomis, 1984 ; Walsh et al., 1988) et se base sur l'évaluation monétaire d'un bien environnemental à partir des résultats d'études antérieures dans des systèmes similaires (Genty, 2006). Elle utilise ainsi l'information et la connaissance existantes sur un ou plusieurs lieux appelés «sites d'étude » ou "sites de référence » vers un autre lieu dit «site d'application» ou «site étudié ».

Deux catégories de MTB existent : i) les transferts de valeurs qui peuvent être une valeur unique, une valeur centrale ou des valeurs à dire d'experts ; ii) les transferts de fonctions qui peuvent être une fonction de demande pour les MCD, une fonction de bénéfice pour les MEC ou un méta-modèle quand il y a plusieurs méthodes utilisées. Plus de détails sur les différents types de méthodes de transfert de bénéfices peuvent être trouvés dans les travaux de Rosenberger et Loomis (2001), Genty (op. cit.) ou de Rakotoarison (op. cit). La littérature existante fait apparaître la supériorité des métamodèles en termes de capacité de prédiction par rapport aux autres MTB (Rosenberger, Loomis, op. cit ; Genty, op. cit).
Il existe de nombreux domaines d'application des MTB au cours de ces trente dernières années. Les activités récréatives en milieu naturel (chasse, randonnée en montagne, pêche...) s'y prêtent particulièrement. Un effort de synthèse important a été mené pour évaluer leur valeur d'usage et de non-usage aux États-Unis et au Canada (Shrestha, Loomis, 2001 ; Rosenberger et Loomis, op. cit. ; Loomis, 2005 ; Loomis, Richardson, 2008). Ces travaux ont abouti à des méta-modèles qui mesurent les variations de bénéfices suivant les types d'activités et des tests de transfert dans ces mêmes pays. Le deuxième domaine d'application des MTB concerne les services écosystémiques. L'évaluation économique de la biodiversité peut se faire soit directement sur les espèces menacées (Loomis, White, 1996) ; soit à travers l'habitat comme les zones humides (Jenkins et al., 2010) . Certains acteurs ont également utilisé les MTB pour évaluer la valeur de la qualité de l'eau (Desvousges et al., 1992 ; Rozan, Stenger, 2000 ; Genty, op.cit. ; Enjolras, Boisson, 2007). De récents travaux (Troy, Wilson, 2006 ; Scolozzi, et al., 2011) montrent cependant que les MTB sur les services écosystémiques doivent s'appuyer sur une analyse spatiale du territoire pour être valables.

L'essor de cette troisième famille de méthodes vient de sa rapidité de mise en œuvre face aux nombreux besoins des gestionnaires dans la prise de décision. Un numéro spécial de la revue Ecological Economics $^{4}$ en 2006 a permis de faire une synthèse sur l'avancement de la recherche sur les MTB. Wilson et Hoehn (2006) notent cependant que l'influence des variables socioéconomiques et environnementales sont complexes dans les transferts de bénéfices, plus particulièrement à l'échelle internationale. Ce papier cherche à mieux analyser ces différents points en prenant les bénéfices de la chasse en Aquitaine comme champ d'application.

6. Ecological Economics, $\mathrm{n}^{\circ} 60$. 


\section{Le modèle théorique de la méta-analyse}

Nous nous basons sur les travaux de Bergstrom et Taylor (2006) pour spécifier la fonction d'utilité indirecte d'un individu qui est de la forme :

$$
U=U(p, R, L, q, S U B, H, I)
$$

où $U$ est la fonction d'utilité indirecte, $p$ le prix des biens marchands à la consommation (nourriture, logement, énergie...), $R$ le revenu du ménage, $L$ les quantités de loisir (nombre de sorties), $q$ la mesure de la qualité des biens non-marchands (présence d'équipement, abondance de gibier, accès...), $S U B$ les quantités de substitut de loisir accessible à l'individu, $H$ les caractéristiques socioéconomiques autres que le revenu (âge, taille du ménage, catégorie socioprofessionnelle...) et enfin $I$ le niveau d'information disponible pour l'individu. Un transfert de la valeur du bénéfice d'un bien nonmarchand noté $C A P$ du site de référence noté $A$ vers un site d'application noté $B$, produira la variation d'utilité suivante :

$$
\begin{aligned}
& \Delta U=U\left(p^{B}, R-C A P, L^{B}, q^{B}, S U B^{B}, H, I\right) \\
& -U\left(p^{A}, R, L^{A}, q^{A}, S U B^{A}, H, I\right)
\end{aligned}
$$

La solution de ce problème conduit à la formalisation du CAP de la manière suivante :

$$
\begin{aligned}
C A P= & f\left(p^{B}-p^{A}, L^{B}-L^{A}, q^{B}-q^{A},\right. \\
& \left.S U B^{B}-S U B^{A}, H, I\right)
\end{aligned}
$$

Si des incertitudes existent sur l'offre et la demande, cette équation comprendra en plus un terme aléatoire. Cette formalisation pose les hypothèses de la méta-analyse. Premièrement, elle montre les conditions d'application de la MTB entre deux sites telles qu'exposées par Rosenberger et Loomis (op. cit.). Les deux sites doivent présenter des caractéristiques communes notamment sur les autres caractéristiques socio-économiques $H$ et les niveaux d'information $I$. Deuxièmement, elle montre que l'effet du revenu s'annule dans la modélisation de l'utilité de l'individu dans l'équation (3).

\section{Les données utilisées}

Nous avons choisi de travailler avec la base de données de l'United States Department of Agriculture (USDA) car elle rassemble les sources les plus significatives en matière d'évaluation de la chasse au grand gibier. Elle a été mobilisée dans plusieurs publications dont notamment Rosenberger et Loomis (op. cit.), Loomis et White (op. cit.) et Genty (2005), ce qui montre sa validité scientifique.

La base de données de l'USDA contient 760 valeurs de bénéfices par jour et par individu provenant de 163 études d'évaluation sur 21 activités récréatives en pleine nature qui ont été réalisées aux États-Unis entre 1967 et 1998. Dans cette base de données, 195 valeurs se rapportent aux bénéfices de la chasse au grand gibier et seront étudiées dans cet article. Nous avons enrichi ces résultats par 65 autres valeurs provenant d'études récentes non citées dans la base de données de l'USDA. Celles-ci couvrent la période 1999-2003 et proviennent à la fois des thèses, des mémoires diffusés sur internet mais également des articles publiés dans Jstor, ScienceDirect et SpringerLink.

Sur l'ensemble des 250 valeurs de la chasse rassemblées, 29 sont issues de journaux scientifiques, 3 des actes de colloque, 50 de travaux universitaires (thèses, mémoires) et 168 de rapports (documents de travail ministériels). Les études ayant eu lieu à des années différentes, les variations temporelles des données ont été corrigées par l'utilisation des taux d'inflation de l'année de l'étude afin de ramener les valeurs en \$ US de l'année 2004'.

Pour chaque estimation, 126 variables $^{8}$ sont renseignées et couvrent les caractéristiques générales de l'étude : (titre, auteurs, date de l'étude, références bibliographiques...), les types de bénéfice (valeurs, changement

7. Le taux d'inflation utilisé provient du site Internet de l'US department of Labor: www.bls.gov.

8. Une description détaillée de ces 126 variables a été publiée, Loomis (op. cit.). 
proposé...), les détails méthodologiques des MEC, les détails méthodologiques des MCD, les descriptions de la zone d'étude (forêt naturelle, parc, lac, privé/publique...) ; les espèces animales présentes sur le milieu, les types d'activité récréative et les variables socioéconomiques de la population (âge, sexe, résidence, revenu moyen...).

L'un des problèmes majeurs de cette base de données est l'insuffisance d'information sur les variables socio-économiques. En effet dans les rapports nationaux provenant des enquêtes quinquennales sur les activités récréatives aux États-Unis, les caractéristiques socio-économiques des enquêtés n'ont pas été détaillées du fait de la taille élevée de l'échantillon (environ 10000 chasseurs). Ce problème a été soulevé dans la plupart des études qui ont utilisé la base de données de l'USDA notamment par Rosenberger et Loomis (op. cit.) et Genty (op. cit). Nous avons essayé d'intégrer les variables socioéconomiques d'un individu moyen de l'année et de l'état où l'étude a été réalisée. Les données sur l'âge moyen, le revenu et le niveau d'éducation aux États-Unis sont accessibles à partir du site www.fedstats.gov. L'influence de ces variables sur les bénéfices de la chasse va être testée dans le métamodèle.

\section{Résultats}

Sur l'ensemble des variables considérées, seules 17 d'entre elles sont significatives et ont été retenues dans le modèle final. Parmi celles-ci, il y a une variable sociale, 4 variables environnementales et 12 variables méthodologiques. Ces variables avec leurs statistiques descriptives sont résumées dans le tableau 1. Concernant les autres variables non retenues, on peut les classer en deux catégories. Premièrement, il y a celles qui sont peu renseignées dans les études (ex : autres variables socio-économiques, qualité des territoires...), ce qui rend difficile l'analyse de leur impact sur les bénéfices de la chasse. Deuxièmement, il y a les variables qui n'ont pas d'influence significative sur les bénéfices de la chasse (ex : localisation des études). Elles restent toutefois utiles à conserver dans la base de données afin de poursuivre le travail de synthèse des études, garantir la traçabilité des valeurs et faciliter la mise à jour ultérieure de la base de données.

Le méta-modèle a été estimé à l'aide d'un modèle économétrique ( $c f$. annexe) basé sur la méthode des Moindres carrées ordinaire (MCO) avec correction de l'auto-correlation et de l'hétéroscedasticité des erreurs par l'estimateur de Newey West. Les détails techniques de la régression sont présentés en annexe. La régression obtenue expliquant les variations des bénéfices de la chasse en 2004 (BEN_2004) est donnée par :

BEN_2004 = 2,67*AGE + 74,00*FORET -
268,39*LAC_RESERVOIR- 103,54*MULTSITE-
49,79*ZONE - 244,70*PRIM + 308,43*BENMES
$-168,05 * \mathrm{MEC}-49,70 *$ EMAIL + 76,29*RECOM
+ 347,64*MECMCO + 76,29*TRONQ +
$220,75 *$ NEG - 62,56*ENCHERE + 127,58*DEP
$-44,77 *$ SUBS + 129,25*MCDMCO

La statistique de Fisher obtenue est significative $(F=1,66 ; p=0,05)$ indiquant qu'il existe un lien important entre la variable dépendante et les variables indépendantes. Son pouvoir explicatif est élevé puisque le coefficient de détermination $\mathrm{R}^{2}$ ajusté est de $80 \%$. D'autres méta-modèles comme ceux de Rosenberger et Loomis (2001) ne dépassent pas les $50 \%$. Ceci montre que le modèle sur la chasse au grand gibier est beaucoup plus robuste que ceux prenant en compte l'ensemble des activités récréatives liées à la nature.

Les résultats détaillés de la régression sont donnés dans le tableau 2 , les variables y sont classées par ordre croissant de significativité. La première colonne donne les abréviations des variables explicatives. $\mathrm{La}$ deuxième colonne désigne les coefficients de chaque variable explicative, la troisième colonne contient les écart-types de ces coefficients. Les résultats du test de Student (1914) sur chaque coefficient sont donnés dans la quatrième et la cinquième colonne. 
Chasse au grand gibier: méthode de transfert des bénéfices

Tableau 1. Statistique descriptive des variables explicatives utilisées dans la méta-analyse

\begin{tabular}{|c|c|c|c|c|c|}
\hline $\begin{array}{c}\text { Nom } \\
\text { des variables }\end{array}$ & Détails et modalités & Médiane & Moyenne & Max & Min \\
\hline \multicolumn{6}{|c|}{ Variable dépendante } \\
\hline BEN_2004 & $\begin{array}{l}\text { Bénéfice de la chasse par jour et par personne } \\
\text { estimé en US\$ en } 2004\end{array}$ & 47,06 & 63,36 & 1118,41 & 1,34 \\
\hline \multicolumn{6}{|c|}{ Variable socio-économique } \\
\hline AGE & $\begin{array}{l}\text { Âge moyen de la population dans chaque état } \\
\text { en années (variable proxy de l'âge des chasseurs) }\end{array}$ & 35,59 & 35,56 & 47 & 27,1 \\
\hline \multicolumn{6}{|c|}{ Variables environnementales } \\
\hline FORET & $\begin{array}{l}\text { Présence de forêt dans le site de récréation } \\
1=\text { oui } ; 0=\text { non } 10,67610\end{array}$ & 1 & 0,676 & 1 & 0 \\
\hline $\begin{array}{l}\text { LACl } \\
\text { RESERVOIR }\end{array}$ & $\begin{array}{l}\text { Présence de lac ou réservoir dans le site } \\
\text { de récréation : } 1=\text { oui ; } 0=\text { non }\end{array}$ & 0 & 0,012 & 1 & 0 \\
\hline MULTSITE & Étude sur plusieurs sites : $1=$ oui ; $0=$ non & 0 & 0,436 & 1 & 0 \\
\hline SUBS & $\begin{array}{l}\text { Inclusion des sites de substitution dans } \\
\text { l'équation de la demande }: 1=\text { oui } ; 0=\text { non }\end{array}$ & 0 & 0,096 & 1 & 0 \\
\hline ZONE & $\begin{array}{l}\text { Zonage aux États-Unis par rapport à leur facilité } \\
\text { d'accés : } 1=\text { zone primaire (entrée limitée) } ; \\
2=\text { zone semi-primitive : zone primaire avec } \\
\text { un accès exclusivement non motorisé } ; \\
3=\text { zone semi-primitive }: \text { zone primaire avec } \\
\text { accès motorisé } ; 4=\text { sentiers naturels, } 5=\text { zone } \\
\text { rurale, } 6=\text { zone urbaine, } 7=\text { milieu varié. }\end{array}$ & 2 & 1,54 & 7 & 0 \\
\hline \multicolumn{6}{|c|}{ Variables méthodologiques } \\
\hline PRIM & $\begin{array}{l}\text { Étude primaire ou secondaire : } \\
1=\text { oui (première utilisation des données) } \\
0=\text { non (réutilisation des données) }\end{array}$ & 0 & 0,488 & 1 & 0 \\
\hline BENMES & Bénéfice mesuré : $1=C A P ; 0=C A R$ & 1 & 0,872 & 1 & 0 \\
\hline MEC & $\begin{array}{l}\text { Évaluation contingente: } 1=\text { oui (MEC) } ; \\
0=\text { autres (MCD, analyse conjointe ou } \\
\text { autres méthodes) }\end{array}$ & 1 & 0,752 & 1 & 0 \\
\hline EMAIL & Enquête par email : 1 = oui ; $0=$ non & 0 & 0,256 & 1 & 0 \\
\hline RECOM & $\begin{array}{l}\text { Recommandation de l'auteur pour un } \\
\text { transfert de bénéfices : } 1=\text { oui } ; 0=\text { non }\end{array}$ & 0 & 0,108 & 1 & 0 \\
\hline \multicolumn{6}{|c|}{ Variables méthodologiques spécifiques aux MEC } \\
\hline MECMCO & $\begin{array}{l}\text { Type de modèle économétrique utilisé : } \\
1=\mathrm{MCO} ; 0=\text { non (MCO semi paramétrique, } \\
\text { Tobit, autres) }\end{array}$ & 0 & 0,004 & 1 & 0 \\
\hline TRONQ & $\begin{array}{l}\text { Existence d'une limite maximale de bénéfices } \\
\text { dans un questionnaire de type ouvert tronquant } \\
\text { ainsi l'échantillon vers le haut : } 1=\text { oui ; } \\
0=\text { pas de limites }\end{array}$ & 0 & 0,008 & 1 & 0 \\
\hline NEG & $\begin{array}{l}\text { Valeurs négatives autorisées dans un } \\
\text { questionnaire de type ouvert : } 1=\text { oui ; } 0=\text { non }\end{array}$ & 0 & 0,012 & 1 & 0 \\
\hline ENCHERE & $\begin{array}{l}\text { Utilisation d'enchère répétitive dans un } \\
\text { questionnaire de type fermé : } \\
1=\text { oui ; } 0=\text { non } 00,01210\end{array}$ & & & & \\
\hline \multicolumn{6}{|c|}{ Variables méthodologiques spécifiques aux MCD } \\
\hline DEP & $\begin{array}{l}\text { Données sur les dépenses rapportées dans } \\
\text { l'étude : } 1=\text { oui; } 0=\text { non }\end{array}$ & 0 & 0,068 & 1 & 0 \\
\hline MCDMCO & $\begin{array}{l}\text { Type de modèle économétrique utilisé : } \\
1=\mathrm{MCO} ; 0=\text { autres ( } \mathrm{MCO} \text { semi paramétrique, } \\
\text { Tobit, poisson, binomiale négative...) }\end{array}$ & 0 & 0,132 & 1 & 0 \\
\hline
\end{tabular}


Les effets des variables socio-économiques, environnementales et méthodologiques sont discutés succinctement.

\section{Impacts des variables socio-économiques sur les bénéfices du grand gibier}

Le coefficient lié à l'âge (AGE) a un signe positif et significatif, ce qui signifie que plus l'individu interrogé est âgé, plus son bénéfice est élevé. Ce résultat traduit le fait que la chasse est une activité récréative qui attire plus les personnes âgées que les jeunes. Ce résultat est confirmé par les travaux de Montgomery et Blalock (2010) et les enquêtes du CSA (2006).

Les autres variables socio-économiques : éducation, revenu du ménage ont été testées mais n'ont pas été significatives. Ceci montre que les bénéfices de la chasse au grand gibier sont assez homogènes au sein des différentes catégories socioprofessionnelles aux États-Unis. Ready et al. (2004) ont également montré que les variables socio-économiques influencent peu la valeur moyenne des bénéfices à travers une MTB menée à l'échelle de différents pays européens.

Spash et Vatn (2006) notent cependant que l'influence des variables socio-économiques dans les MTB n'est pas encore suffisamment claire. Leur non-significativité peut être due aux lissages des variations des bénéfices dans les rapports et donc, dans les bases de données servant à mettre en place les MTB. C'est le cas notamment des études référencées dans la base de données de l'USDA qui viennent en grande partie des enquêtes nationales quinquennales qui concernent environ 10000 chasseurs. Dans celles-ci, les distances entre le lieu de chasse et le lieu de résidence, les caractéristiques socio-économiques et les profils des chasseurs ne sont pas donnés. Cette non-significativité peut également être liée à la non-pertinence des variables proxy utilisées pour substituer les données socio-économiques manquantes. En effet, nous avons testé les caractéristiques moyennes de la population dans chaque état où l'étude a eu lieu. Des données à une échelle plus fine pourrait apporter plus de précision.

Tableau 2. Résultat de la méta-analyse sur la chasse au grand gibier après une correction de l'autocorrelation et de l'hétéroscedasticité des erreurs

\begin{tabular}{l|c|c|c|c}
\hline Variable & Coefficient & Écart type & $t$ de Student & Probabilité \\
\hline MECMCO & 347,65 & 47,7 & 7,29 & 0,00 \\
\hline FORET & 74 & 10,84 & 6,83 & 0,00 \\
\hline NEG & 220,75 & 32,72 & 6,75 & 0,00 \\
\hline MEC & $-168,05$ & 26,49 & $-6,34$ & 0,00 \\
\hline BENMES & 308,44 & 49,84 & 6,19 & 0,00 \\
\hline MULTSITE & $-103,54$ & 17,02 & $-6,08$ & 0,00 \\
\hline ENCHERE & $-62,57$ & 10,32 & $-6,06$ & 0,00 \\
\hline PRIM & $-244,7$ & 40,86 & $-5,99$ & 0,00 \\
\hline ZONE & $-49,79$ & 8,53 & $-5,84$ & 0,00 \\
\hline DEP & 127,59 & 22,6 & 5,65 & 0,00 \\
\hline EMAIL & $-49,71$ & 12,66 & $-3,93$ & 0,00 \\
\hline RECOM & 76,3 & 21,42 & 3,56 & 0,00 \\
\hline MCDMCO & 129,26 & 44,96 & 2,87 & 0,00 \\
\hline LAC_RESERVOIR & $-268,4$ & 95,63 & $-2,81$ & 0,01 \\
\hline TRONQ & 76,29 & 29,48 & 2,59 & 0,01 \\
\hline SUBS & $-44,77$ & 18,03 & $-2,48$ & 0,01 \\
\hline AGE & 2,68 & 1,34 & 2,01 & 0,05 \\
\hline
\end{tabular}




\section{Impacts des variables environnementales}

Les valeurs des bénéfices de la chasse sont plus élevées dans les zones forestières (FORET) et plus faibles au bord des lacs et des réservoirs (LAC/RESERVOIR). Les sites de chasse présentant un taux de couverture forestière sont donc les plus valorisés par les chasseurs de grand gibier car la densité de la population animale y est généralement plus élevée. Par ailleurs, nous nous sommes intéressés plus particulièrement au grand gibier et non au gibier d'eau, ce qui explique le signe négatif de la variable LAC/RESERVOIR.

Les différents types de zonage de l'occupation du sol aux États-Unis (ZONE) ont également une influence sur les bénéfices des chasseurs. Les zones primitives sont moins accessibles à la pratique de la chasse au grand gibier que les zones rurales. En effet, le zonage actuel aux États-Unis autorise la pratique de la chasse dans les différentes catégories de zones mais la pratique des activités de loisir est plus restreinte dans les zones primitives. Montgomery et Blalock (op. cit.) confirment dans leur étude que l'accès au territoire de chasse est une des contraintes majeures les plus mentionnées par les chasseurs aux États-Unis. Lindhjem (2007) montre également que les bénéfices des biens et services non-marchands sont moins élevés dans les forêts avec une forte protection. Plus les zones sont donc considérées comme accessibles, plus les bénéfices sont élevés.

Les variables MULTISITE et SUBS présentent des coefficients négatifs, on peut en déduire que si l'étude considère la possibilité de fréquenter d'autres sites, les bénéfices trouvés sont plus faibles. L'insertion des sites de substitution dans la fonction de demande a pour effet de diminuer les bénéfices mesurés. Carson et al. (1996) ont également trouvé le même résultat en comparant les MCD menés sur plusieurs sites avec celles menées sur un site. Ce résultat est également similaire à ceux de Loomis et Rosenberger (op. cit.) et Shrestha et Loomis (op. cit.) mais inverse à celui d'Enjolras et Boisson (op. cit.).

\section{Impacts des choix méthodologiques}

La plupart des signes des coefficients liés aux variables méthodologiques correspondent aux résultats trouvés dans d'autres méta-modèles. La variable MEC indiquant qu'il s'agit d'une évaluation contingente possède un signe négatif, ce qui signifie que les méthodes de constat de préférence donnent des bénéfices plus faibles que les méthodes de révélation des préférences. Comme nous l'avons expliqué dans la présentation de ces méthodes, ce résultat est dû à la différence de type de fonctions de demande entre les deux familles de méthodes et aux échantillons d'individus interrogés dans les MEC qui contiennent des non-usagers donnant des bénéfices généralement faibles ou nuls. Ce résultat peut également provenir du fait que les MCD sont basées sur des comportements réels et que les MEC sont fortement dépendantes des scénarios proposés dans l'enquête. Ceci correspond aux résultats trouvés par Walsh et al. (1992), Carson et al. (op. cit.), Loomis et Rosenberger (op. cit), Genty (2005), Enjolras et Boisson (op. cit.) et Loomis et Richardson (op. cit).

Concernant le type de modélisation économétrique, il apparaît que les modèles de type MCO donnent des bénéfices plus élevés que ceux qui ont été estimés avec les modèles non linéaires (probit, logit, Tobit, négative binomiale, etc.). Cette remarque est valable aussi bien pour l'évaluation contingente (MECMCO) que pour le coût de déplacement (MCDMCO). Ceci vient du fait que les MCO ne tiennent pas compte des non-réponses et du caractère aléatoire des réponses (Adamowicz et al., 1989). La forme fonctionnelle choisie par les auteurs a donc des conséquences non négligeables sur le calcul du bénéfice comme l'a également montré Rosenberger et Loomis (op. cit.) lors de la construction des méta-modèles aux États-Unis. 
Des consignes provenant de l'enquêteur dans le cadre d'une MEC avec un questionnaire de type ouvert semblent augmenter les bénéfices prononcés par l'individu. C'est le cas même s'il s'agit de prononcer une limite maximale aux bénéfices (TRONQ) ou de donner la possibilité d'annoncer une valeur négative (NEG) dans un questionnaire ouvert. Bohara et al. (2001) expliquent que les CAP négatifs sont liés en général à une spécification économétrique de MCO et qu'ils sont dus à la présence de personnes opposées aux changements proposés par le scénario. Lorsqu'on réinterroge l'individu sur ses propres réponses avec des enchères répétitives (ENCHERE) dans un questionnaire fermé, les bénéfices tendent à diminuer. Ce résultat a également été démontré par Rosenberger et Loomis (op. cit.).

Par ailleurs, lorsque les dépenses des individus (ex : hôtel, restauration, équipement, etc.) sont rapportées clairement dans l'étude (DEP), on remarque que les bénéfices sont plus élevés. Ceci est contre intuitif mais il peut s'agir d'un indicateur de qualité des études fournissant ces données qui ont aidé les répondants à mieux répondre aux enquêtes.

Le coefficient de la variable BENMES indiquant si l'étude est basée sur une estimation du CAP et celle du CAR, a un signe positif dans la régression. Or, plusieurs études dont Bishop et Heberlein (1979), Knetsch et Sinden (1987), ont montré que les CAP étaient 3 à 15 fois inférieurs aux CAR. Ce résultat contradictoire à la littérature peut provenir de plusieurs sources. Premièrement, le nombre des études mesurant le CAR dans la base de données est faible, ce qui laisse penser à une moins bonne connaissance des études utilisant cette mesure. Deuxièmement, la compa- raison de ces deux types de surplus est difficile car les valeurs obtenues dépendent de l'importance du changement proposé sur les pratiques des usagers (changement de qualité, augmentation du plan de chasse...). Enfin, il peut s'agir d'une erreur de codification lors de la construction de la base de données. Nous avons pu le vérifier en reprenant certains documents originaux comme celui de Loomis, Creel et Cooper (1988).

Le mode d'enquête utilisé (EMAIL) semble également avoir une influence sur les valeurs de bénéfices trouvés. Nos résultats montrent que les enquêtes par email donnent des valeurs plus faibles que les enquêtes sur site, par courrier ou par téléphone. Ceci est lié au fait que les enquêtes sur site permettent en général de mieux cibler les usagers qui ont une meilleure connaissance de la ressource, en l'occurrence le grand gibier dans cette étude. Une enquête par email présente néanmoins l'avantage de toucher à la fois des usagers et des nonusagers de la ressource mais les bénéfices estimés sont plus variables. Ce résultat a également été noté par plusieurs auteurs (Boyle, 2003 ; Lindhjem, op. cit.).

Les études primaires donnent également des valeurs plus faibles par rapport aux études secondaires (PRIM) puisque ces dernières se basent généralement sur des données agrégées qui ne présentent plus de valeurs extrêmes et donc peu de variation. La variable RECOM indiquant que l'étude est recommandée par les auteurs pour faire une MTB a un signe positif. Par ailleurs, la variable indiquant si l'évaluation contingente a été menée avec une question ouverte ou fermée n'est pas significative. Il n'y a donc pas de tendance particulière dans les études sur la chasse au grand gibier concernant cette variable contrairement aux travaux de Genty (op. cit.). 


\section{Le transfert du méta-modèle avec la Région Aquitaine}

\section{L'ajustement des conditions de chasse}

Pour effectuer le transfert du méta-modèle vers un site d'application, toutes les variables explicatives doivent être remplacées par les propres valeurs. Compte tenu du nombre élevé des variables méthodologiques dans la régression, seules trois variables explicatives peuvent être ajustées au cas de la Région Aquitaine.

- AGE : c'est l'âge moyen de la population par état utilisé pour remplacer l'âge des chasseurs. L'âge moyen dans la Région Aquitaine en France est de 41 ans (source : Institut national de la statistique et des études économiques Insee).

- FORET : c'est la variable indiquant si la chasse se déroule dans une zone forestière ou non. Dans la Région Aquitaine, les zones de chasse sont multiples. En faisant l'hypothèse que les territoires de chasse sont répartis de façon homogène dans toute la région, on peut utiliser le pourcentage de zones boisées qui est de $48 \%$ selon les données fournies par l'Institut français de l'environnement (Ifen, 2005) dans la base cartographique Corine Land Cover en 2000.

- LAC_RESERVOIR : c'est la variable indiquant la présence de rivières ou réservoirs d'eau dans le site de chasse. Elle peut également être traitée de la même manière que la variable FORET. Les lacs représentent moins de $1 \%$ du territoire dans la Région Aquitaine (Ifen, op. cit.).

En ce qui concerne les variables liées aux méthodes ainsi que celles non observables sur le site d'intérêt, Rosenberger et Loomis (op. cit.) recommandent d'utiliser la valeur moyenne trouvée dans les études de référence. Avec ces adaptations, nos estimations donnent un bénéfice moyen de 65,36 US\$/jour/personne en 2004.

\section{La prise en compte des différences de taux de change}

Pour convertir ce bénéfice en euros, il faut utiliser l'indice de Parité du pouvoir d'achat (PPA) mis en place par l'OCDE. En effet, le taux de change d'une monnaie par rapport à une autre ne reflète que leurs valeurs réciproques sur les marchés financiers internationaux et non leurs valeurs intrinsèques pour un consommateur. Le PPA est un critère qui synthétise les différences de situation économique et donc de différence de pouvoir d'achat dans différents pays. Avec un indice du PPA de 1 US\$ équivalent à $0,94 €$ en 2004, la valeur moyenne du bénéfice en Aquitaine est donc estimée à $61,44 € /$ jour/personne avec une intervalle de confiance à $95 \%$ de 50,11 € et 72,77 €. L'utilisation d'une MEC donnera un bénéfice de 21,53 € tandis qu'une MCD conduit à une valeur de $122,41 €$. L'ensemble des résultats est repris dans le tableau 3.

Tableau 3. Résultats des estimations du bénéfice de la chasse dans la Région Aquitaine avec I'application du PPA

\begin{tabular}{l|c|c|c}
\hline & Valeur moyenne & $\begin{array}{c}\text { Valeur moyenne } \\
\text { avec MEC }\end{array}$ & $\begin{array}{c}\text { Valeur moyenne } \\
\text { avec MCD }\end{array}$ \\
\hline Bénéfice estimé en US\$ 2004 & 65,36 & 22,90 & 190,96 \\
\hline Borne supérieure en US\$ & 77,41 & 34,95 & 203,01 \\
\hline Borne inférieure en US\$ & 53,31 & 10,85 & 178,91 \\
\hline PPA entre la France et les États-Unis & $\$ 0,94$ & & \\
\hline Bénéfice estimé en $€$ 2004 & 61,44 & 21,53 & 122,41 \\
\hline Borne supérieure en $€$ & 72,77 & 32,86 & 130,13 \\
\hline Borne inférieure en $€$ & 50,11 & 10,20 & 114,68 \\
\hline
\end{tabular}




\section{Test de validité du transfert de bénéfices}

Comme Navrud (2006), Rosenberger et Loomis (op. cit.), nous avons utilisé une technique de validation externe pour valider et vérifier la fiabilité de la MTB. Il s'agit d'un test d'hypothèse global de comparaison entre les valeurs originales et celles obtenues par la MTB sur le site d'application. Navrud (op. cit.) propose le test d'erreur noté $T E$ suivant :

$T E=\left|\frac{B E N_{T}-B E N_{R}}{B E N_{T}}\right|$

$B E N_{P}$ représente la « vraie » valeur du bénéfice (CAP ou CAR) estimée avec les méthodes classiques comme les MEC ou MCD ; $B E N_{T}$ la valeur du bénéfice pour le site étudié estimée avec la MTB. À ce jour, il n'existe pas d'étude primaire sur le bénéfice de la chasse dans la Région Aquitaine ce qui rend difficile l'application du test de validité externe. Pour substituer une étude primaire, nous allons nous baser sur les enquêtes nationales sur les dépenses des chasseurs révélées par Pinet (1987), Vollet et Bretière (2009). En moyenne, le bénéfice trouvé est de $27,3 €$ pour une sortie de chasse. Si on utilise cette valeur comme étant le $B E N_{P}$ dans la formule (4), on obtient un taux d'erreur de $18 \%$ avec la MEC ; $300 \%$ avec la MCD et $108 \%$ toutes méthodes confondues. Shrestha et Loomis (op. cit.) trouvent des taux d'erreur d'environ $28 \%$ en utilisant une MTB à l'échelle internationale. La moyenne dans le cadre des transferts de bénéfices est située entre 25 et $40 \%$ (Navrud, op. cit. ; Ready et Navrud, 2006). Le taux d'erreur ne semble donc être acceptable, dans notre cas, que pour la MEC.

\section{Discussions et conclusion}

Cet article montre que les MTB permettent de trouver des valeurs de référence à un bien ou un service non-marchand sans nécessiter de mettre en place des protocoles d'études lourds et coûteux. On peut conclure que le développement des tech- niques de transferts de bénéfices constitue une avancée prometteuse pour aider à la prise de décision en matière de recherche de gestion durable. Le travail sur la méta-analyse donne quelques pistes d'actions à mener pour mieux gérer cette ressource naturelle : retenir la population de chasseurs expérimentés qui valorisent le plus la ressource, encourager le recrutement des jeunes chasseurs par des systèmes de prix et de formation, revoir les montants des cotisations cynégétiques, réduire les zones non accessibles à la chasse (ex : réserve biologique intégrale), améliorer l'accès à la chasse dans les forêts qui constituent à la fois des refuges pour le gibier et des territoires de chasse très appréciés. L'analyse de l'ensemble des bénéfices non-marchands et des coûts du grand gibier avec la dynamique de la population devra par la suite aboutir à une réflexion plus approfondie sur la mise en place d'une politique de gestion durable de la chasse. On peut également utiliser le bénéfice trouvé pour faire une ACB. Si on fait la somme des bénéfices de la chasse dans la Région Aquitaine, avec environ 10000 chasseurs de grand gibier sortant en moyenne 35 fois par $a^{9}$ et un bénéfice de $61,44 €$ par personne et par sortie, on peut estimer à environ 21,5 millions d'euros le bénéfice total annuel de la chasse. Ce résultat semble dominer les coûts engendrés par le grand gibier en milieu agricole et forestier qui se chiffrent au minimum à 2 millions d'euros par an (Rakotoarison, op. cit.). D'autres types de coûts engendrés par le grand gibier ont cependant été peu étudiés dans la Région Aquitaine jusqu'ici : les dégâts forestiers en forêt mixte (zones du Périgord, du pays de l'Adour et du massif Pyrénéen), les coûts

9. Le nombre moyen de sorties de chasse en France est estimé 25 par an selon Pinet (1987). Dans le SudOuest, où il s'agit d'une activité assez populaire, il peut être estimé en moyenne à 35 par an si on reprend les données de l'enquête menée par le CSA en 2006. 
des collisions avec les automobiles, les risques sanitaires ${ }^{10}$ ainsi que les effets des ongulés sur la biodiversité.

Cette analyse souligne trois éléments importants à prendre à compte dans la mise en œuvre des MTB :

(i) la diversité des méthodes d'évaluation et les effets des choix méthodologiques sur les bénéfices d'une ressource naturelle ;

(ii) la qualité des données utilisées ;

(iii) les différences socio-économiques entre les sites de référence et le site d'application.

Premièrement, les résultats de la métaanalyse montrent que les coefficients liés aux variables méthodologiques sont largement plus élevés en valeur absolue que ceux des variables socio-économiques et environnementales. Ceci signifie que pour une même ressource naturelle, les bénéfices nonmarchands estimés vont surtout dépendre de la manière dont on les estime et non pas de leurs caractéristiques intrinsèques, ni des caractéristiques de la population qui en fait usage. Ce point est important à prendre en compte puisque les résultats d'une évaluation économique vont servir de base de discussions à la prise de décision. Il est nécessaire que les économistes qui travaillent sur ces questions trouvent un consensus et avancent dans une amélioration des méthodes.

Deuxièmement, la méta-analyse est conditionnée par la pertinence des données utilisées. Certaines variables liées spécifiquement à la chasse ne sont pas renseignées dans la base de données de l'USDA. Plusieurs auteurs (Loomis, Creel, Cooper, op. cit. ; Vollet, Bretière, op. cit.) ont cependant souligné l'importance d'autres variables environnementales (richesse faunistique,

10. Le grand gibier peut transmettre des maladies à l'homme (maladie de Lyme) ou aux animaux domestiques. qualité des territoires, proximité au lieu d'habitation, etc.), juridiques (loi chasse, régime des associations de chasse, cotisation, date des chasses...) et socio-économiques (revenu, niveau d'éducation, culture, etc.). Elles peuvent avoir un effet important sur la valeur des bénéfices mais n'ont pas pu être intégrées dans cette étude. Par ailleurs, l'inexistence de méthode d'évaluation similaire sur la Région Aquitaine rend difficile la validation de la MTB. Nous rejoignons les conclusions de Loomis et Rosenberger (op. cit) sur la nécessité d'améliorer la qualité des informations mises dans la restitution d'une étude primaire et dans les bases de données servant à mettre en œuvre les MTB.

Troisièmement, on peut se poser des questions sur les conditions de transfert des bénéfices à l'échelle internationale. Dans notre cas, il y a des points communs et des différences sur les caractéristiques socioéconomiques du site d'application (Région Aquitaine) et des sites de référence (ÉtatsUnis). D'abord, les proportions de chasseurs sur la population totale sont relativement proches : $5 \%$ aux États-Unis et 2,38\% en France ${ }^{11}$. Ensuite, dans les deux sites, on observe la baisse du nombre de chasseurs : $1 \%$ par an aux États-Unis contre $2 \%$ par an dans la Région Aquitaine. Puis, la proportion de femmes chasseurs est faible dans les deux sites : $7 \%$ aux États-Unis et $2 \%$ en France. Enfin, l'écart de revenu annuel moyen des chasseurs est relativement faible : $23841,81 €$ aux États-Unis ${ }^{12}$ contre $21792,95 €$ dans la Région Aquitaine ${ }^{13}$.

11. Les chiffres sur les chasseurs dans la Région Aquitaine se basent sur l'étude du Conseil sondage analyses (CSA) en 2006 tandis que ceux des chasseurs aux États-Unis du rapport de l'U.S. Department of Fish and Wildlife Service et al. (2006). 12. 31400 US\$ par an, soit $23841,81 €$ aux ÉtatsUnis avec un taux de change 1,37 US $\$$ /€ donné par le FMI en 2006. 
Ceci rend le transfert de bénéfices possible entre les deux sites.

Cependant, les chasseurs français sont plus âgés que les chasseurs américains : 55 ans en moyenne en France contre 44 ans aux États-Unis. Cette différence a pu être corrigée avec l'aide du méta-modèle qui a été créé. La France possède dix fois moins de chasseurs que les États-Unis : 1,4 million contre 15,23 millions. Il s'agit donc d'une activité plus courante aux États-Unis qu'en France. Par ailleurs, la différence qui est la plus difficile à surmonter dans le cadre d'un transfert de bénéfice international est relative aux préférences des agents économiques.
Le nombre annuel moyen de sorties de chasse en France est de 25, contre 35 dans la région du Sud-Ouest et 14 aux ÉtatsUnis. Or, la moyenne des dépenses annuelles des chasseurs est de $1590 €$ en France contre $390 €^{14}$ aux États-Unis. Ces deux derniers points montrent que la préférence individuelle des chasseurs français pour cette activité est beaucoup plus prononcée que celle des chasseurs américains. Les bénéfices de la chasse mesurés avec les MTB ne remplacent pas les valeurs issues d'une étude primaire mais ils donnent un ordre de grandeur de la valeur de cette activité dans la culture régionale.
13. Calcul suivant les catégories socioprofessionnelles des chasseurs français selon l'enquête du CSA 2006, et recoupé avec le salaire moyen en Aquitaine selon l'Insee.
14. Dépenses des chasseurs aux États-Unis = 534 US\$, soit environ $390 €$ par an avec un taux de change donné par la FMI 1 euro $=1,37 \$$ en 2006. 


\section{RÉFÉRENCES BIBLIOGRAPHIQUES}

Adamowicz W.-L., Fletcher J.-J., GrahamTomasi T. (1989). Functional form and the statistical properties of welfare measures. American Journal of Agricultural Economics, vol. 71, n 2, p. 414-421.

Bergstrom J., Taylor L. (2006). Using metaanalysis for benefits transfer: Theory and practice. Ecological Economics, vol. 60, $\mathrm{n}^{\circ} 2$, p. 351-360.

Bishop R., Heberlein T. (1979). Measuring Values of Extramarket Goods: Are Direct Measures Biased? American Journal of Agricultural Economics, ${ }^{\circ}$ 61, p. 926-930.

Bohara A., Kerkvliet J., Berrens R. (2001). Addressing Negative Willingness to Pay in Dichotomous Choice Contingent Valuation. Environmental \& Resource Economics, vol. 20, n ${ }^{\circ}$, p. 173-195.

Bonnieux F., Desaigues B. (1998). Économie et politiques de l'environnement. Paris, Dalloz, 328 p.

Boyle K. (2003). Contingent valuation in practice. In «A primer on nonmarket valuation», Boston, Kluwer Academic Publishers, p. 111-170.

Carson R.-T., Flores N.-E., Martin K.-M., Wright J.-L. (1996). Contingent valuation and revealed preference methodologies: Comparing the estimates for quasi-public goods. Land Economics, vol. 72, ${ }^{\circ} 1, \mathrm{p}$. 80-99.

Ciriacy-Wantrup (1947). Capital Returns from Soil Conservation Practices. Journal of Farms Economics, ${ }^{\circ} 29$, p. 11801190.

CSA (2006). Les Chasseurs qui sont-ils? http://www.chasseurdefrance.com/presentation/frame.php?page $=6$ (vue le 05 juillet 2011).

Cummings R.-G., Brookshire D.-S., Schultz W.-D. (1986). Valuing Environmental Goods:An Assessment of the Contingent Valuation Method. Totowa, Rowman \& Allanheld Publishers, 270 p. Desvousges W.-H., Naughton M.-C.,
Parsons G.R. (1992). Benefit transfer conceptual problems in estimating waterquality benefits using existing studies. Water Resources Research, vol. 28, $\mathrm{n}^{\circ} 3$, p. 675-683.

Enjolras G., Boisson J.-M. (2007). Valuing lagoons using a meta-analytical approach: Methodological and practical issues. Thessalonique, conférence annuelle de l'EAERE, 27-30 juin, p. 1-24.

FNC (2006). Base de données sur le nombre des chasseurs en Aquitaine. Communication personnelle.

Genty A. (2005). Du concept à la fiabilité de la méthode du transfert en économie de l'environnement : un état de l'art. Cahiers d'économie et sociologie rurales, $\mathrm{n}^{\circ} 77$, p. 5-34.

Genty A. (2006). Méthode de transfert et calculs économiques : application dans le domaine de l'eau. Paris, Thèse de doctorat en Sciences économiques, Université Paris 1, $390 \mathrm{p}$.

Greene W. (2002). LIMDEP version 8.0, Econometric Modeling Guide. Volume 2, Econometric Software, Inc. Plainview, NY, USA, 714 p.

Hotelling H. (1949). Letter to the National Park Service. In United States National Parks Service (ed.), «An economic study of the monetary valuation of recreation in the national parks», Washington DC, Land and Recreational Planning Division, National Park Service.

Ifen (2005). Base de données d'occupation $d u$ sol nationale datée 2000. www.stats.environnement.developpement-durable.gouv.fr/.../CORINE_ Land_Cover_-_Condition_Utilisation.htm (vu le 5 juillet 2011).

Jenkins W.-A., Murray B.-C., Kramer R.-A., Faulkner S. (2010). Valuing ecosystem services from wetlands restoration in the Mississippi Alluvial Valley. Ecological Economics, vol. 69, n 5, p. 1051-1061. 
Knetsch J.-L., Sinden J.-A. (1987). The Persistence of Evaluation Disparities. Quarterly Journal of Economics, p. 691-695.

Krutilla J. (1967). Conservation Reconsidered. The American Economic Review, vol. $57 \mathrm{n}^{\circ} 4$, p. 777-786.

Lindhjem H. (2007). 20 years of stated preference valuation of non-timber benefits from Fennoscandian forests: A metaanalysis. Journal of Forest Economics, vol. $12, \mathrm{n}^{\circ} 4$, p. 251-277.

Loomis J. (2005). Updated outdoor recreation use values on national forests and other public lands. Portland, USDA Forest Service Pacific Northwest Research Station, 39 p.

Loomis J.-B., Rosenberger R.-S. (2006). Reducing barriers in future benefit transfers: Needed improvements in primary study design and reporting. Ecological Economics, vol. 60, $\mathrm{n}^{\circ} 2$, p. 343-350.

Loomis J., Richardson L. (2008). Technical documentation of benefit transfer and visitor use estimating models of wildlife recreation, species and habitats. Washington DC, Defenders of Wildlife, $45 \mathrm{p}$.

Loomis J.-B., Creel M., Cooper J. (1988). Economic Benefits of Deer in California: Hunting and Viewing Values. Davis, CA, University of California, Institute of Ecology Report, 332 p.

Loomis J.-B., White D.-S. (1996). Economic benefits of rare and endangered species: summary and meta-analysis. Ecological Economics, vol. 18, ${ }^{\circ} 3$, p. 197-206. Montgomery R., Blalock G. (2010). The impact of access, cost, demographics, and individual constraints, on hunting frequency and future participation. Academy of Marketing Studies Journal, $\mathrm{n}^{\circ} 2$, p. 5-18.

Navrud S. (2006). Benefit Transfer Protocol, Nancy. Workshop on Protocol and Database for Environmental Valuation of French/European Forests, 26-27 Septembre.

Newey W.-K., West K.-D. (1987). A Simple,
Positive Semi-Definite, Heteroskedasticity and Autocorrelation Consistent Covariance Matrix. Econometrica, vol. 55, p. 703-708.

ONCFS, FNC (2010). Tableau de chasse ongulés sauvages saison 2009-2010 « cervidés ». Supplément Faune Sauvage, vol. $289, \mathrm{n}^{\circ} 4$, p. 1-8.

Pinet J.-M. (1987). L'économie de la chasse. Paris, Comité national d'information chasse-nature, $96 \mathrm{p}$.

Rakotoarison H. (2009). Analyse et modélisation de la gestion du grand gibier : cas de la région Aquitaine. Thèse de doctorat ès sciences économiques, Université Montesquieu - Bordeaux IV, 390 p.

Ready R., Navrud S. (2006). International benefit transfer: Methods and validity tests. Ecological Economics, ${ }^{\circ} 60$, p. 429-434.

Ready R., Navrud S., Day B., Dubourg R., Machado F., Mourato S., Spanninks F., Rodriquez M.X.V. (2004). Benefit transfer in Europe: how reliable are transfers between countries? Environmental and Resource Economics, $\mathrm{n}^{\circ} 29$, p. 67-82.

Rosenberger R.-S., Loomis J.-B. (2001). Benefit transfer of outdoor recreation use studies: A technical document supporting the Forest Service Strategic Plan (2000 revision). Fort Collins, CO, USDA Forest Service Rocky Mountain Research Station, $64 \mathrm{p}$.

Rozan A., Stenger A., 2000. Intérêts et limites de la méthode du transfert de bénéfices. Économie et Statistique, vol. 336, n 6, p. 69-77.

Saint-Andrieux C. (2005). Estimation $d u$ coût économique des collisions véhiculesongulés. Paris, ONCFS OGE, 90 p.

Scherrer V. (2002). Réinventer la chasse pour le XXI siècle. Paris, Éd. des Journaux Officiels. Avis et rapports du conseil économique et social, $215 \mathrm{p}$.

Scolozzi R., Morri E., Santolini R. (2011). Delphi-based change assessment in ecosystem service values to support strategic spatial planning in Italian land- 
scapes. Ecological Indicators. Article en presse.

Shrestha R.-K., Loomis J.B. (2001). Testing a meta-analysis model for benefit transfer in international outdoor recreation. Ecological Economics, vol. 39, ${ }^{\circ}$ 1, p. 67-83.

Sorg C.-F., Loomis J.-B. (1984). Empirical Estimates of Amenity Forest Values: A Comparative Review, Fort Collins, CO: US Department of Agriculture, Rocky Mountain Forest and Range Experiment Station, $23 \mathrm{p}$.

Spash C.-L., Vatn A. (2006). Transferring environmental value estimates: Issues and alternatives. Ecological Economics, vol. $60, \mathrm{n}^{\circ} 2$, p. 379-388.

Student (1914). The elimination of spurious correlation due to position in time or space. Biometrika, $\mathrm{n}^{\circ}$ 5, p. 351-360.

Troy A., Wilson M.-A. (2006). Mapping ecosystem services: Practical challenges and opportunities in linking GIS and value transfer. Ecological Economics, vol. $60, \mathrm{n}^{\circ} 2$, p. 435-449.
U.S. Department of Fish and Wildlife Service, the Interior and U.S. Department of Commerce, U.S. Census Bureau (2006). National Survey of Fishing, Hunting, and Wildlife-Associated Recreation, $168 \mathrm{p}$.

Vollet D., Bretière G. (2009). La chasse : Quel impact économique sur l'emploi local ? Une première approche à partir de quelques études de cas. In Colloque SFER "Chasse, territoires et développement durable : outils d'analyse, enjeux et perspectives » Clermont-Ferrand, le 25 et 26 mars, p. 1-23.

Walsh R.-G., Johnson D.-M., McKean J.-R. (1988). Review of Outdoor Recreation Demand Studies with Nonmarket Benefit Estimates. Fort Collins, Water Resource Research Institute Colorado State University, 1968-1988.

Wilson M.-A., Hoehn J. (2006). Valuing environmental goods and services using benefit transfer: The state-of-the art and science. Ecological Economics, vol. 60, $\mathrm{n}^{\circ} 2$, p. 335-342. 


\section{ANNEXE \\ Présentation du modèle économétrique}

Le modèle est estimé à partir d'une forme d'utilité linéaire qui reste la plus utilisée dans les méta-analyses (Rosenberger, Loomis, op. cit.). La régression se présente de la façon suivante :

$y_{i}=\beta_{1} X_{1 i}+\beta_{2} X_{2 i}+\beta_{3} X_{3 i}+\varepsilon_{i}$

où $i$ fait référence à l'identification des valeurs trouvées dans la littérature, $y_{i}$ la variable dépendante qui est le CAP/CAR par jour et par individu, $X_{1 i}$ un vecteur regroupant les variables liées à la méthodologie utilisées dans l'étude pour évaluer l'activité de chasse, $X_{2 i}$ le vecteur des variables socio-économiques (âge, éducation, revenu...) et $X_{3 i}$ le vecteur des variables liées aux qualités des sites (type des milieux, aménagement...). $\beta_{j}$ sont les paramètres estimés des $j$ variables et $\varepsilon_{i}$ représente les erreurs d'estimation du modèle.

Le test de White est significatif avec $F=7,64$ et $p=0,00$, ce qui montre qu'un modèle de régression simple obtenu avec les MCO présente une autocorrélation et une hétéroscedascitité des erreurs. Ces problèmes peuvent être liés aux variables explicatives, qui présentent les mêmes modalités sur plusieurs observations : mêmes méthodes d'évaluation, mêmes régions donc mêmes variables socio-économiques par l'usage des variables proxy. Ceci a pour conséquence de biaiser la variance des coefficients obtenus avec l'estimateur MCO simple et de fausser les autres tests d'hypothèse du modèle.

La correction de la régression linéaire simple se fait avec l'estimateur de Newey West HAC (Heteroskedasticity and Autocorrelation Consistent) (Newey et West, 1987) qui est donné par :

$\sum_{N W}=\left(X^{\prime} X\right)^{-1} \times \sum_{t=1}^{T} e_{t}^{2} x_{t} x_{t}^{\prime} \times\left(X^{\prime} X\right)^{-1}$

$+\left(X^{\prime} X\right)^{-1} \times\left\{\frac{1}{T} \sum_{j=1}^{L} \sum_{t=j+1}^{T}\left(1-\frac{j}{L+1}\right) e_{t} e_{t-j}\left[x_{t} x_{t-j}{ }^{\prime}+x_{t-j} x_{t}^{1^{\prime}}\right]\right\} \times\left(X^{\prime} X\right)$

où $T$ représente le nombre total d'observations et $e_{t}$ est le résidu du modèle basé sur les MCO. $L$ est un paramètre qui indique le nombre de décalage pour calculer les autocorrélations dans les résidus (Greene, 2002). 